\title{
Comprendre la spectroscopie infrarouge : principes et mise en œuvre
}

Les vibrations moléculaires sont à l'origine de l'absorption du rayonnement infrarouge (IR) par la matière, car les niveaux d'énergie moléculaires vibrationnels sont séparés par des énergies qui tombent dans le domaine infrarouge du spectre électromagnétique. La partie infrarouge du rayonnement électromagnétique est partagée en trois domaines : le proche infrarouge (le plus énergétique) qui s'étend de 14000 à $4000 \mathrm{~cm}^{-1}(0,7-2,5 \mu \mathrm{m}$ en longueurs d'onde) ; l'infrarouge moyen qui va de 4000 à $400 \mathrm{~cm}^{-1}(2,5-25 \mu \mathrm{m})$ et enfin l'infrarouge lointain, qui couvre le domaine spectral de 400 à $10 \mathrm{~cm}^{-1}$ (25-1000 $\left.\mu \mathrm{m}\right)$. La mise en œuvre de l'interaction d'un rayonnement infrarouge avec un échantillon, puis la détection et l'analyse spectrale (par transmission ou par réflexion) de ce rayonnement après qu'il ait interagi avec la matière est l'objet de la spectroscopie infrarouge. Cette spectroscopie, très sélective, est couramment utilisée pour l'identification de composés mais elle permet également d'obtenir des informations très importantes sur les interactions inter- et/ou intra-moléculaires, sur la conformation des molécules, sur l'organisation de la matière...

\section{Laurent SERVANT, \\ Gwenaëlle LE BOURDON, \\ Thierry BUFFETEAU}

Université Bordeaux 1

Institut des sciences moléculaires

Groupe de spectroscopie moléculaire

I.servant@ism.u-bordeaux1.fr

\section{Fréquence de vibration et activité}

Les atomes constitutifs de la matière sont liés entre eux et vibrent avec une fréquence liée à leur masse et à la force des liaisons chimiques dans lesquelles ils sont engagés. Une approximation simple et utile permet de comparer la fréquence de vibration $v$ d'une molécule diatomique $A-B$ à celle d'un oscillateur harmonique idéal liant les deux masses $m_{A}$ et $m_{B}$ (des atomes $\mathrm{A}$ et $\mathrm{B})$ à ses extrémités :

$$
v=\frac{1}{2 \pi} \sqrt{\frac{\mathrm{k}}{\mu}}
$$

où $\mu=m_{\mathrm{A}} \cdot m_{\mathrm{B}} /\left(m_{\mathrm{A}}+m_{\mathrm{B}}\right)$ est la masse réduite de l'oscillateur et $k$, la constante de raideur du ressort qui caractérise la force de la liaison chimique. Une telle relation est approximative car elle ne tient pas compte de la nature quantique de la liaison chimique, mais elle permet cependant d'apprécier simplement certains effets sur les spectres de vibration :

- les bandes d'absorption sont situées plutôt vers les basses fréquences quand des atomes lourds sont impliqués;

- la constante de force étant sensiblement proportionnelle à la nature des liaisons (simple, double ou triple), la fréquence associée à la vibration d'une liaison éthylénique est environ $\sqrt{2}$ fois plus élevée que celle associée à une simple liaison carbone-carbone.

Si on reprend l'analogie de la vibration moléculaire avec un oscillateur, la mécanique quantique prévoit que l'énergie vibrationnelle $E$ d'un oscillateur harmonique de fréquence propre $v$ est quantifiée et dépend d'un nombre quantique de vibration $\vee(V=0,1,2, \ldots)$ :

$$
E=h v(V+1 / 2)
$$

Quand la fréquence du rayonnement incident est identique à une fréquence possible de la vibration de la liaison, il pourra y avoir absorption de l'énergie transportée par l'onde incidente. Cette condition est nécessaire mais pas suffisante ! Pour qu'il y ait absorption du rayonnement, il faut qu'en plus la vibration considérée produise une modification de la distribution des charges électriques entraînant une variation du moment dipolaire électrique de la molécule (règle de sélection). Plus précisément, il y aura absorption du rayonnement infrarouge si le produit scalaire du champ électromagnétique local (celui de I'onde incidente et des champs induits) avec le moment dipolaire électrique induit au cours de la vibration est non nul. Ainsi, la molécule $\mathrm{O}_{2}$ est dite inactive en infrarouge : cela ne signifie pas qu'elle ne vibre pas! C'est juste que son mouvement de vibration, selon l'axe de la liaison, n'induit pas de variation du moment dipolaire, et ne peut donc pas être " détecté " par spectroscopie infrarouge. Par contre, $\mathrm{HCl}$ est active en infrarouge, de même que $\mathrm{CH}_{4}$ (bien que cette molécule n'ait pas de moment dipolaire intrinsèque, la vibration d'une liaison $\mathrm{C}-\mathrm{H}$ induit une modification instantanée de son moment dipolaire). Cette règle de sélection est très importante et peut révéler de précieuses informations sur la symétrie d'une molécule et/ou de son environnement. Par exemple, si au cours de la vibration d'une molécule isolée la modification "globale » du moment dipolaire est nulle, il n'y aura pas d'absorption infrarouge ; à l'inverse, l'interaction d'une molécule avec une autre molécule, ou avec une surface (en catalyse, par exemple) peut modifier la variation du moment dipolaire et faire apparaître de nouvelles bandes d'absorption, caractéristiques des interactions subies.

La spectroscopie infrarouge apparaît donc comme une technique susceptible de fournir des informations sur la structure et la composition chimique des échantillons étudiés. 
Après avoir discuté les propriétés des vibrations moléculaires, nous présenterons plusieurs configurations expérimentales courantes pour la mise en œuvre de la spectroscopie infrarouge selon la nature des échantillons étudiés (liquides, films, poudres...) : par transmission, par réflexion (spectroscopie de réflexion-absorption infrarouge (IRRAS), réflexion totale atténuée (ATR), réflexion diffuse (DRIFT)).

\section{Vibrations moléculaires}

Une vibration moléculaire - ou un mode de vibration - correspond à un mouvement périodique, de fréquence $v$, de chacun des atomes constitutifs de la molécule. Nous allons nous intéresser maintenant au nombre de modes de vibration attendus pour une molécule isolée formée de $N$ atomes. Chacun des atomes peut bouger selon trois directions de l'espace, ce qui correspond, au total, à $3 N$ mouvements possibles. Puisque nous nous intéressons exclusivement aux vibrations, il faut soustraire à ces mouvements ceux qui correspondent à des déplacements de translation de la molécule (trois degrés de liberté) et à des mouvements de rotation (trois degrés de liberté). Finalement, $3 \mathrm{~N}-6$ déplacements peuvent être considérés comme des modes de vibration ( $3 N-5$ pour des molécules linéaires). Il apparaît donc clairement que l'analyse des spectres infrarouges (on parle « d'attribution vibrationnelle » lorsqu'on peut corréler chaque bande d'absorption avec une vibration moléculaire (comme indiqué sur le tableau 1) devient rapidement inextricable quand les molécules possèdent un nombre important d'atomes. Afin de simplifier l'attribution des spectres, il est d'usage de distinguer parmi les vibrations moléculaires, les mouvements d'élongation, notés $v$ (les longueurs des liaisons varient mais les angles restent les mêmes) et les mouvements de déformation, notés $\delta$ (les longueurs des liaisons restent les mêmes mais les angles varient). Souvent, cette distinction n'est qu'une approximation car les deux types de mouvements se combinent pour un même mode !

\begin{tabular}{|c|c|c|}
\hline Vibrations & Domaine spectral $\left(\mathrm{cm}^{-1}\right)$ & Intensité IR observée \\
\hline$v(\mathrm{O}-\mathrm{H})$ & $3650-3000$ & v \\
\hline$v(\mathrm{~N}-\mathrm{H})$ & $3500-3300$ & $\mathrm{~m}$ \\
\hline$v(\equiv \mathrm{C}-\mathrm{H})$ & 3300 & $\mathrm{~F}$ \\
\hline$v(=\mathrm{C}-\mathrm{H})$ & $3100-3000$ & $\mathrm{~m}$ \\
\hline$v(-\mathrm{C}-\mathrm{H})$ & $3000-2800$ & $\mathrm{~F}$ \\
\hline$v(\mathrm{C} \equiv \mathrm{N})$ & $2255-2220$ & $\mathrm{~F}$ \\
\hline$v(C \equiv C)$ & $2250-2100$ & $f$ \\
\hline$v(C=0)$ & $1820-1680$ & TF \\
\hline$v(\mathrm{C}=\mathrm{N})$ & $1680-1610$ & $\mathrm{~m}$ \\
\hline$v(\mathrm{C}=\mathrm{C})$ & $1675-1600$ & $\mathrm{~m}$ \\
\hline$\delta(\mathrm{N}-\mathrm{H})$ & $1650-1500$ & $\mathrm{~F}$ \\
\hline$v(\mathrm{C}=\mathrm{C})$ aromatique & $1620-1450$ & v \\
\hline$v(\mathrm{~N}=\mathrm{N})$ azo & $1450-1400$ & $f$ \\
\hline$\delta\left(\mathrm{CH}_{2}\right), \delta a(\mathrm{CH} 3)$ & $1480-1400$ & $\mathrm{~m}$ \\
\hline$\delta_{s}(\mathrm{CH} 3)$ & 1380 & $\mathrm{~F}-\mathrm{m}$ \\
\hline$v(C-C)$ & $1300-800$ & $m-f$ \\
\hline$v(\mathrm{C}-\mathrm{Cl})$ & $750-600$ & $\mathrm{~F}$ \\
\hline$v(\mathrm{C}-\mathrm{Br})$ & $650-500$ & $\mathrm{~F}$ \\
\hline$v(C-I)$ & $600-450$ & $\mathrm{~F}$ \\
\hline
\end{tabular}

TF : très forte, $F$ : forte, $v$ : variable, $m$ : moyenne, $f$ : faible

Tableau 1. Fréquences et intensités observées pour divers modes de vibration.

À l'exception de l'état gazeux sous faible pression (où les molécules sont suffisamment éloignées les unes des autres), les oscillateurs moléculaires peuvent interagir entre eux et se coupler. II est alors possible d'observer des déplacements en fréquence des modes observés à l'état liquide ou solide par comparaison à ceux obtenus à l'état gazeux pour la molécule à l'état isolé. Par exemple, dans le cas d'un cristal, on distingue des « vibrations internes", très voisines de celles observées dans le spectre du composé étudié à l'état liquide ou gazeux et des «vibrations de réseau ", à très basse fréquence (typiquement 100-250 $\mathrm{cm}^{-1}$ ) caractéristiques de la symétrie du motif cristallin.

\section{Exploitation des spectres}

L'analyse des spectres infrarouges obtenus en transmission et en réflexion (diffuse, ATR ou spéculaire) nécessite idéalement d'extraire les signaux de grandeurs communes et intrinsèques aux échantillons qui permettent une interprétation la plus quantitative possible. Lorsqu'une onde électromagnétique pénètre dans un milieu, elle subit des modifications liées à sa fréquence, à son état de polarisation et à la nature du milieu traversé. Dans le cas où la réflexion est négligea- ble, la transmission $T$ du rayonnement subit une décroissance exponentielle en fonction de l'épaisseur $d$ traversée :

$$
\mathrm{T}(\bar{v})=\exp [-\alpha(\bar{v}) \cdot d]
$$

où $\alpha(\bar{v})$ représente le coefficient d'absorption de l'échantillon. Le coefficient d'absorption est relié au coefficient d'extinction $\mathrm{k}(\bar{v})$ qui est la partie imaginaire de l'indice de réfraction complexe de l'échantillon $(\tilde{n}(\bar{v})=n(\bar{v})+i k(\bar{v}))$ par la relation:

$$
\alpha(\bar{v})=4 \pi \mathrm{k}(\bar{v}) \bar{v}
$$

En solution, le coefficient d'absorption $\alpha(\bar{v})$ est souvent remplacé par le produit $\varepsilon(\bar{v}) \cdot C$, où $\varepsilon(\bar{v})$ représente le coefficient d'extinction molaire de la molécule étudiée à la fréquence considérée et $C$ la concentration de la solution.

Il est d'usage de calculer l'absorbance (ou densité optique $\mathrm{DO}), \mathrm{A}(\overline{\mathrm{v}})$ définie par :

$$
A(\bar{v})=-\log [T(\bar{v})]=\frac{\alpha(\bar{v}) \cdot d}{\operatorname{Ln}(10)}=\frac{\varepsilon(\bar{v}) \cdot C \cdot d}{\operatorname{Ln}(10)}
$$

Cette dernière relation traduit la loi de Beer-Lambert, valable pour des films solides pas trop absorbants ou des solutions diluées ; elle prédit la proportionnalité de l'absorbance $\mathrm{A}(\overline{\mathrm{v}})$ avec l'épaisseur et le coefficient d'extinction pour un solide et avec la concentration, le trajet optique et le coefficient d'extinction molaire pour une solution absorbante. 
Plus généralement, ce sont les constantes optiques (indice de réfraction $\mathrm{n}(\bar{v})$ et coefficient d'extinction $\mathrm{k}(\bar{v})$ ) qui permettent de remonter aux propriétés intrinsèques de la matière. En effet, les spectres $\mathrm{n}(\bar{v})$ et $\mathrm{k}(\overline{\mathrm{v}})$ caractérisent complètement les propriétés spectroscopiques des échantillons étudiés contrairement aux spectres de réflexion et de transmission dont l'allure et l'intensité dépendent de la quantité de matière et des conditions expérimentales utilisées. Les domaines de fréquence où $\mathrm{k}(\bar{v})$ est non nul correspondent à des bandes d'absorption de l'échantillon, qui se traduisent donc par des absorbances non nulles. Il faut cependant bien avoir à l'esprit que dans le domaine de fréquence où $\mathrm{k}(\bar{v})$ est non nul, $n(\bar{v})$ a lui aussi un comportement singulier. En effet, on sait classiquement que loin des bandes d'absorption, l'indice de réfraction décroît avec la longueur d'onde (on parle de dispersion normale) ; par contre, au voisinage d'une bande d'absorption (donc, typiquement sur le domaine spectral où $\mathrm{k}(\bar{v})$ est non nul), I'indice de réfraction croît avec la longueur d'onde (on parle de dispersion anormale), ce qui donne une allure caractéristique du comportement en fréquence des constantes optiques. Dans le cas des mesures en transmission, le signal obtenu dépend essentiellement de $\mathrm{k}(\bar{v})$ alors qu'il dépend de $n(\bar{v})$ et de $\mathrm{k}(\bar{v})$ dans les mesures en réflexion. La détermination des constantes optiques peut être réalisée à partir du spectre de réflexion spéculaire sous incidence quasi normale pour un matériau massique ou à partir du spectre ATR polarisé perpendiculairement au plan d'incidence pour une couche mince déposée sur le cristal ATR. La détermination des constantes optiques à partir d'un seul spectre expérimental peut être réalisée car les dépendances fréquentielles de $\mathrm{n}(\bar{v})$ et $\mathrm{k}(\bar{v})$ sont reliées par les relations de KramersKronig.

\section{Appareillage et enregistrement des spectres}

Les sources de rayonnement infrarouge sont généralement constituées par des solides (barreau de $\mathrm{SiC}$ ) portés à haute température (1500 K) qui rayonnent par incandescence. Les détecteurs de rayonnement infrarouge sont thermiques ou quantiques. Les détecteurs thermiques DTGS (sulfate de triglycine deutériée) sont couramment utilisés car ils permettent de faire des mesures de la transmittance ou de la réflectance très précises (en absolu), mais ils sont beaucoup moins sensibles que les détecteurs quantiques ( $\mathrm{MCT}, \mathrm{InSb}$ ). Les détecteurs quantiques MCT (alliage de mercure, cadmium et tellure) sont souvent utilisés lorsque le signal mesuré est très faible (microscopie IR par exemple) mais ils ne sont généralement pas linéaires. À l'heure actuelle, la plupart des spectromètres infrarouges utilise un interféromètre de Michelson (constitué d'un miroir mobile, d'un miroir fixe et d'une séparatrice) positionné entre la source et le détecteur. Le rayonnement IR traverse l'interféromètre et on enregistre les variations de luminosité du faisceau sortant de l'interféromètre en fonction du déplacement du miroir mobile : c'est l'interférogramme. Le spectromètre est équipé d'un calculateur qui permet de calculer la transformée de Fourier de l'interférogramme pour aboutir au spectre infrarouge (spectre simple faisceau). Classiquement, un premier spectre $\mathrm{I}_{0}(\bar{v})$ est enregistré sans échantillon (spectre simple faisceau de référence ou «background ») ; puis le spectre de l'échantillon

$\mathrm{I}(\bar{v})$ est enregistré.
Le spectre de transmittance $\mathrm{T}(\overline{\mathrm{v}})=\frac{\mathrm{I}(\overline{\mathrm{v}})}{\mathrm{I}_{0}(\bar{v})}$ est ensuite calculé en faisant le rapport des deux spectres simples faisceaux.

\section{Échantillonnage et techniques d'analyse}

La préparation de l'échantillon pour l'enregistrement de son spectre infrarouge est une étape cruciale qui dépend de son état physique et de la quantité de matière disponible. Clairement, l'enregistrement

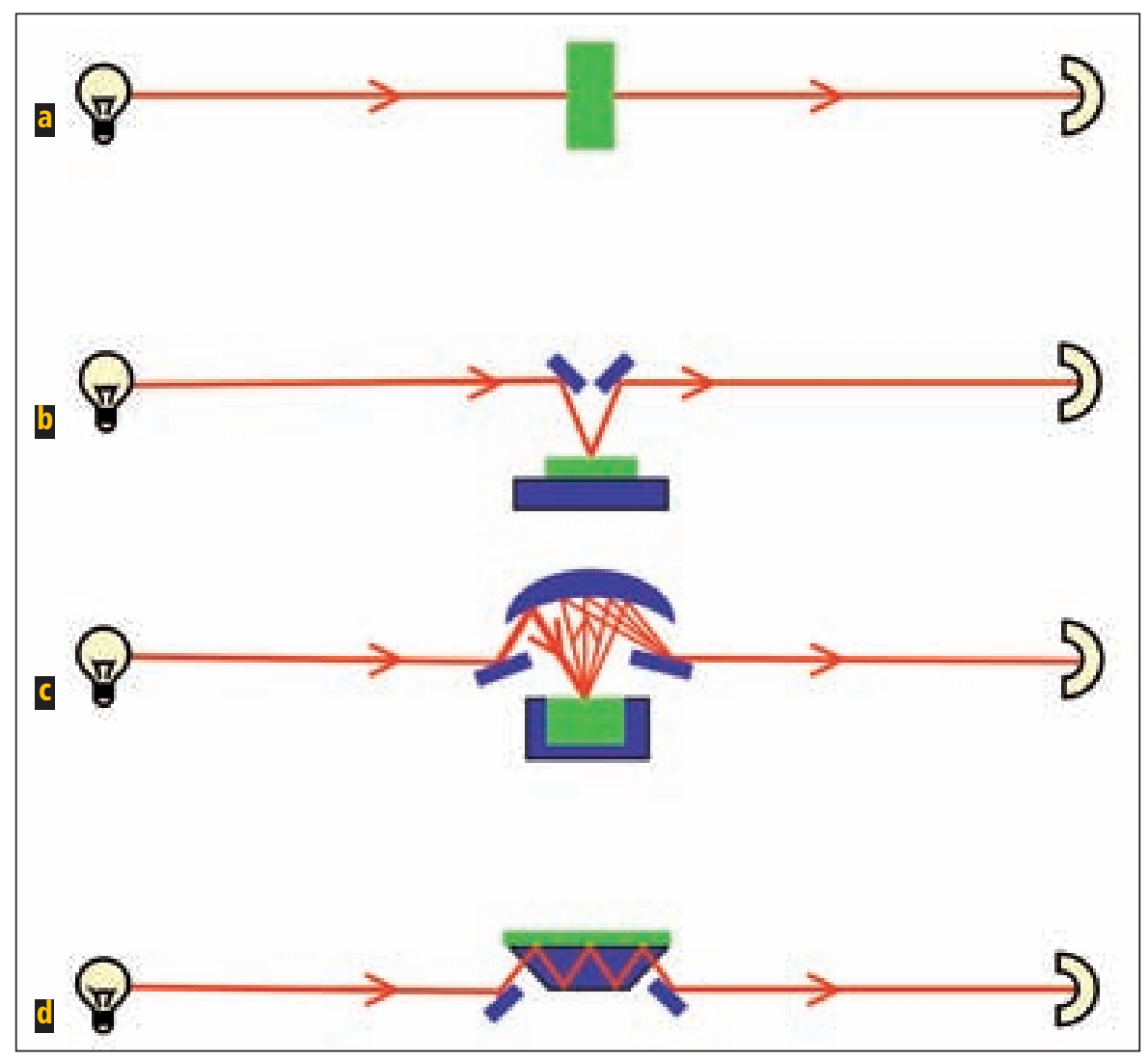

Figure 1. Accessoires courants pour l'échantillonnage en spectroscopie infrarouge : a. montage en transmission (par défaut) ; b. montage en réflexion spéculaire ; $c$. montage de réflexion diffuse (échantillons massifs dont la surface est rugueuse, échantillons pulvérulents...) ; $d$. montage de réflexion totale atténuée (ATR) : un prisme de fort indice de réfraction dans I'IR est au contact d'un échantillon. 
d'un spectre d'un échantillon massif, pulvérulent, liquide ou sous forme de film mince... $n$ 'impose pas les mêmes contraintes ! Une exigence commune cependant à ces situations est que le signal infrarouge enregistré soit suffisamment intense pour être exploitable. Par exemple, on conçoit que le spectre en transmission d'un film ultramince de quelques dizaines de nanomètres soit très peu intense et difficile à exploiter : c'est pour cela que des approches expérimentales très diverses (figure 1) ont été développées et sont désormais disponibles sous forme d'accessoires adaptables dans les compartiments échantillon des spectromètres commerciaux.

\section{Analyse par transmission}

La manière la plus simple d'enregistrer le spectre IR d'un échantillon est de procéder par transmission lorsque l'échantillon n'absorbe pas totalement le rayonne-

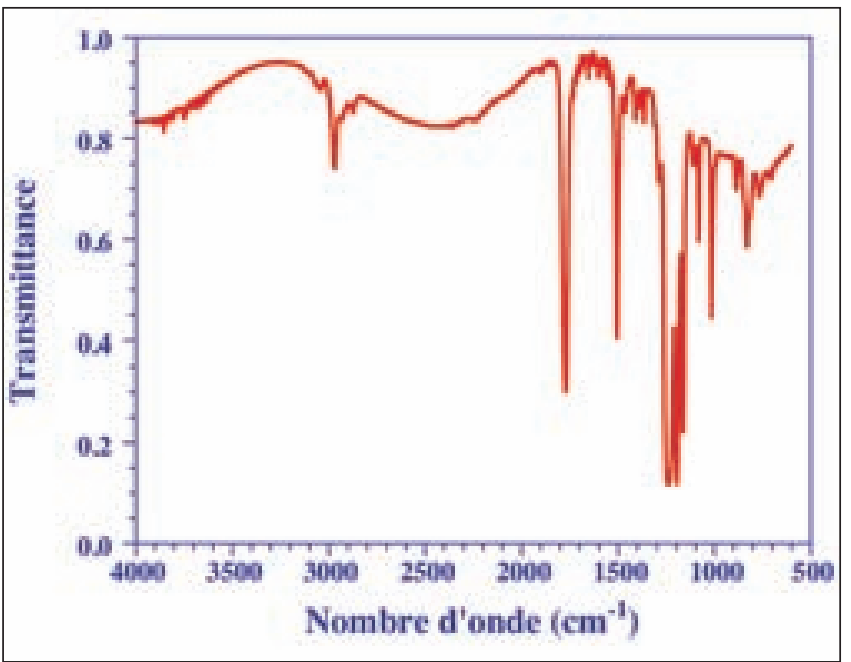

$\mathrm{CO}_{2}$ ). En outre, cette technique permet d'étudier des couches minces déposées sur des substrats transparents au rayonnement infrarouge (figure 2), mais également des molécules en solution dans des solvants organiques ou aqueux. Dans ce dernier cas, le spectre IR de la molécule étudiée sera obtenu après soustraction du spectre IR du solvant utilisé. La qualité du spectre IR sera conditionnée par le choix des conditions expérimentales (trajet optique,

Figure 2. Spectre de transmittance d'un film de polycarbonate de $2 \mu \mathrm{m}$ d'épaisseur (l'ondulation de la ligne de base est une structure $\mathrm{d}^{\prime}$ interférence de pas $\Delta \bar{v}=\frac{1}{2 \text { nd }}$ ).

ment dans le domaine spectral d'intérêt (cf. paragraphe précédent). Le rapport des deux spectres simples faisceaux $\mathrm{I}(\bar{v})$ et $\mathrm{I}_{0}(\bar{v})$ permet l'élimination des bandes d'absorption de l'environnement (par exemple, celles de la vapeur d'eau et du concentration de la solution, nature des fenêtres de la cellule).

\section{Analyse par réflexion spéculaire externe}

Quand l'échantillon est trop absorbant, aucun rayonnement $n^{\prime}$ est transmis et nous sommes contraints de recourir à des techniques de réflexion. Les montages couramment utilisés (figure 1) conservent la configuration du compartiment

\section{OPTOELECTRONIOUE}

\section{La Flexibilité et I'Expertise au service de I'Innovation}

- Emetteurs UV-VIS-IR

LEDs et Power LEDs

Solutions "Chip on Board"

Sources lumineuses à LED ou Xénon

Diodes lasers

Lampes flash Xénon

Corps noirs miniatures

- Détecteurs UV-VIS-IR

Pyrodétecteurs,

Thermopiles,

Photodiodes,

Phototransistors,

Photorésistances.

SPCM, Compteurs de photons.

Photomultiplicateur CPM.

Photodiodes PIN et APD.

Tubes images.
HTDS sera present NDP 2011 / $4-8$ jullet / Lyon - Coogits StP 148 jullet I Bondeaus.

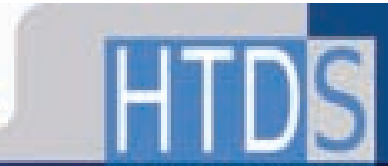

APFLICATIONS
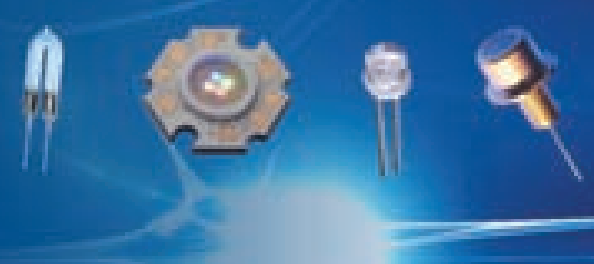

* industrieflas

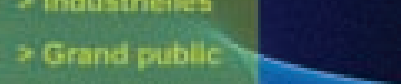

7 Medicales

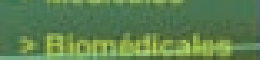

$\rightarrow$ Aderonguntiques
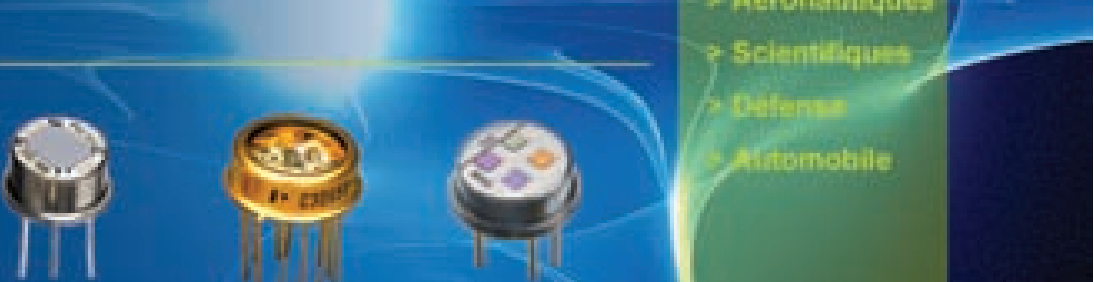

Des solutions at un sarvies sur mesurs 
échantillon du spectromètre et permettent l'enregistrement d'un spectre de réflexion à partir d'une modification adaptée du trajet du faisceau. Si l'état de surface de l'échantillon le permet (rugosité de surface très inférieure à la longueur d'onde du rayonnement IR), un accessoire de réflexion spéculaire externe peut être utilisé (figure 1b). Les accessoires utilisés sont optimisés de manière à rediriger le faisceau IR incident vers le détecteur grâce à un jeu de miroirs (l'angle d'incidence peut être fixe ou variable). Les spectres de réflexion spéculaire de matériaux massiques (la réflexion provient uniquement de la première interface air/matériau) présentent généralement des bandes ayant l'allure de dispersions reflétant les variations spectrales des constantes optiques (figure 3a). Un traite- ment particulier du spectre (analyse de Kramers-Kronig) est nécessaire pour remonter au coefficient d'extinction et à l'indice de réfraction du matériau afin de déterminer les fréquences des différentes vibrations (figures $3 c$ et $3 d$ ) : on peut remarquer la similarité entre le spectre du coefficient d'extinction (figure $3 d$ ) et le spectre d'absorbance obtenu à partir d'un film de polycarbonate de $2 \mu \mathrm{m}$ d'épaisseur (figure 3b). Dans le cas de couches minces déposées sur divers substrats, les bandes d'absorption de l'échantillon pourront apparaître positivement ou négativement par rapport à la ligne de base en fonction des conditions expérimentales utilisées (angle d'incidence, polarisation de la lumière et propriétés optiques du substrat utilisé). Notons finalement que I'utilisation de la lumière polarisée parallèlement au plan d'incidence sous incidence rasante permet d'obtenir les spectres de réflexion de couches ultraminces déposées sur des surfaces métalliques (spectroscopie IRRAS).

\section{Analyse par réflexion diffuse}

$\mathrm{Si}$, au contraire, la rugosité de surface est de l'ordre de la longueur d'onde du rayonnement incident (typiquement de l'ordre du micromètre), comme c'est le cas pour des surfaces rugueuses ou des matières pulvérulentes, alors l'énergie du faisceau réfléchi n'est plus concentrée dans une seule direction (celle de la réflexion spéculaire). Elle est, au contraire, dispersée dans toutes les directions de l'espace par des effets de diffraction, réfraction et réflexion, et on parlera de réflexion diffuse. La détection du rayon-
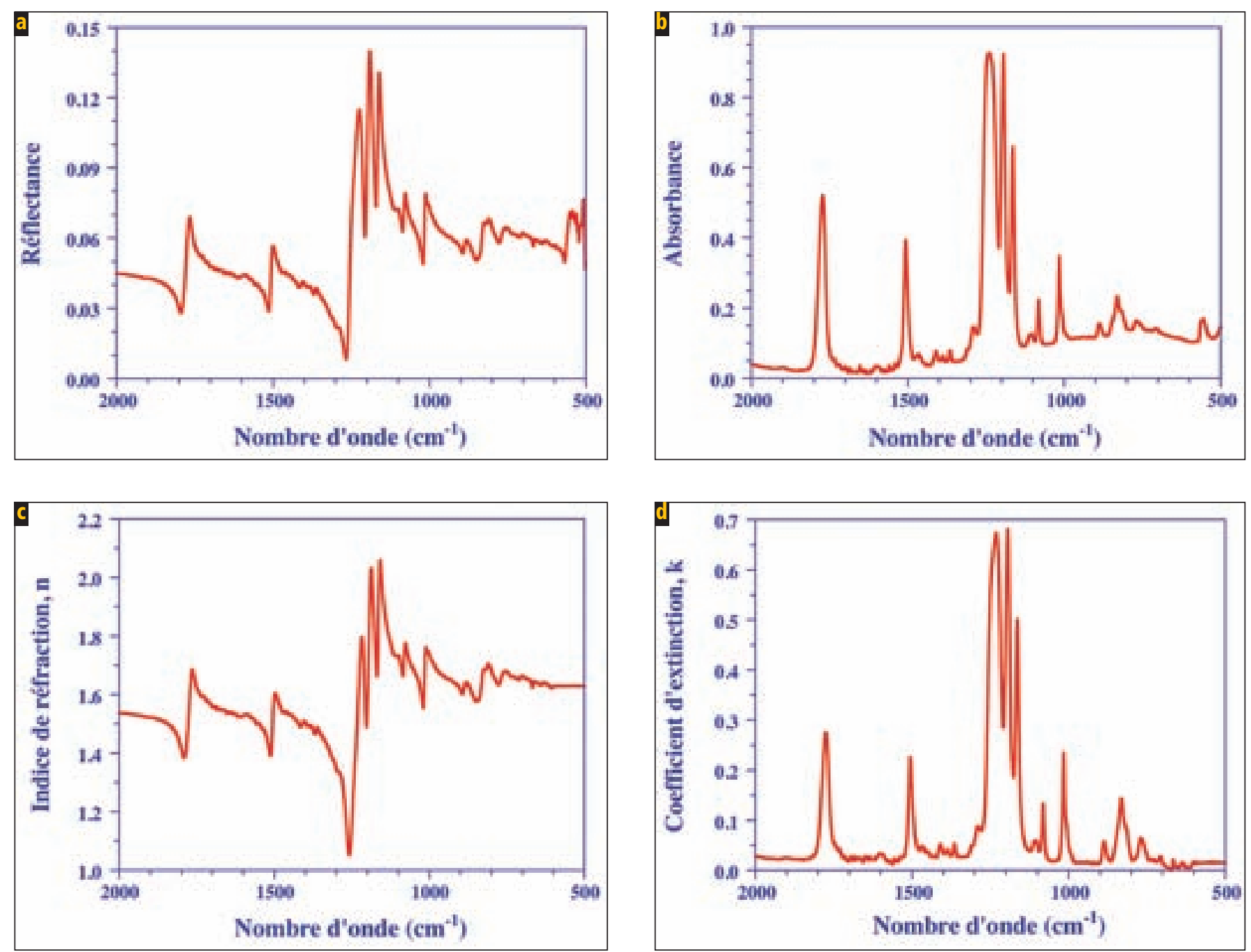

Figure 3. a. Spectre de réflectance spéculaire externe (angle d'incidence de $12^{\circ}$ ) d'un échantillon massique de polycarbonate; $b$. Spectre d'absorbance d'un film de polycarbonate de $2 \mu \mathrm{m}$ d'épaisseur ; c. Indice de réfraction $\mathrm{n}(\bar{v})$ et $d$. coefficient d'extinction $\mathrm{k}(\bar{v})$ du polycarbonate, calculés par analyse de Kramers-Kronig du spectre de réflectance spéculaire externe. 


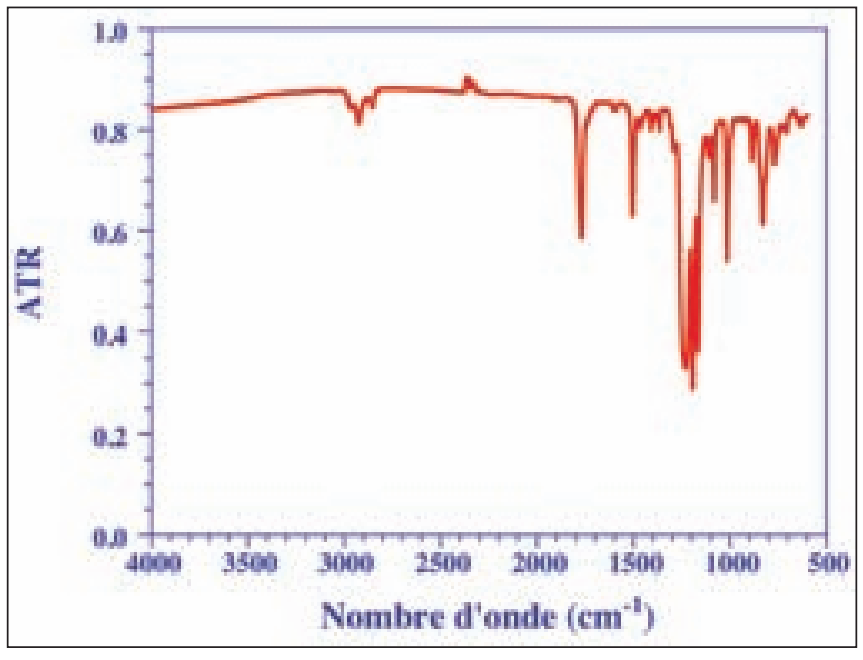

Figure 4. Spectre de réflectance totale atténuée d'un film de polycarbonate de $2 \mu \mathrm{m}$ d'épaisseur.

(germanium, par exemple). Le cristal servira de guide d'onde au rayonnement et permettra à l'onde évanescente d'interagir en nement réfléchi nécessite dans ce cas un accessoire spécifique : l'échantillon est surmonté par un dôme réflecteur hémisphérique qui collecte l'ensemble du rayonnement issu de l'échantillon, et le focalise vers le détecteur. Là encore, il est nécessaire d'extraire du spectre de réflexion diffuse une grandeur intrinsèque qui caractérise l'absorption du rayonnement par l'échantillon diffusant : c'est l'objectif du traitement de Kubelka et Munk qui permet d'obtenir un coefficient d'absorption effectif d'un échantillon diffusant à partir de son spectre de réflexion diffuse.

\section{Analyse par réflexion totale atténuée}

Une autre méthode d'enregistrement des spectres infrarouge repose sur la mise en œuvre des conditions de réflexion totale. Quand un rayon lumineux se propage dans un milieu d'indice de réfraction $n_{1}$ supérieur à l'indice $n_{2}$ de l'échantillon à analyser, il y aura réflexion totale à l'interface qui sépare les deux milieux (absence de rayonnement réfracté) pour une valeur de l'angle d'incidence $\theta_{i}$ supérieure à un angle critique $\theta_{c}$, telle que $\sin \theta_{c}=\frac{n_{2}}{n_{1}}$.

En fait, bien que l'on dise que la réflexion soit totale, une onde évanescente pénètre néanmoins dans l'échantillon sur une distance de l'ordre du micromètre. Cette approche expérimentale, appelée réflectance totale atténuée (ATR), peut être mise en œuvre en plaçant l'échantillon à analyser au contact d'un cristal à fort indice de réfraction dans l'infrarouge surface (quelques milliers d'Angströms pour un cristal de germanium) avec l'échantillon au contact du cristal, et d'obtenir ainsi son spectre infrarouge. Une telle démarche permet d'enregistrer les spectres IR de liquides (même absorbants), de poudres, de pâtes et de films souples (figure 4).

\section{Glossaire des termes fréquemment rencontrés en spectroscopie infrarouge}

ATR : Attenuated Total Reflectance DRIFTS : Diffuse Reflectance Infrared Fourier Transform Spectroscopy

IR : Infrared

IRRAS : Infrared Reflection Absorption Spectroscopy

FT-IR : Fourier Transform Infrared Spectroscopy

KK : Kramers-Kronig

KM : Kubelka-Munk

RAIRS : Reflection-absorption infrared spectroscopy

TIRS : Transmission Infrared Spectroscopy

VCD : Vibrational Circular Dichroism

\section{Pour en savoir plus}

IR Spectroscopy. An Introduction, par Helmut Günzler et Hans-Ulrich Gremlich, Wiley-VCH, 2002. Remarquable ouvrage d'initiation très détaillé sur les principes de la spectroscopie infrarouge, les méthodes modernes d'échantillonnage, l'interprétation des spectres (analyse qualitative; quantitative).

Handbook of Vibrational Spectroscopy, Volumes 1 à 5 , 2002. Cet ouvrage est très complet et rassemble de nombreuses informations sur l'utilisation des spectroscopies vibrationnelles dans des domaines très variés. Éditeurs : J. M. Chalmers et P. R. Griffiths, John Wiley \& Sons,

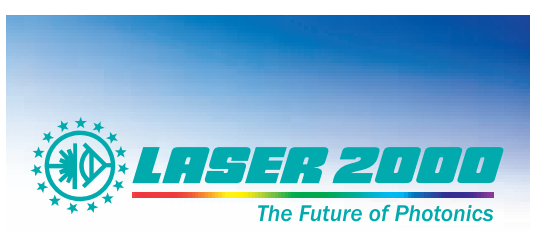

\section{Lasers \& Sources de lumière}

- Lasers à solide

- Lasers à gaz

- Lasers et amplificateurs à fibre

- Diodes lasers composants

- Modules diodes lasers

- Sources de lumière large bande

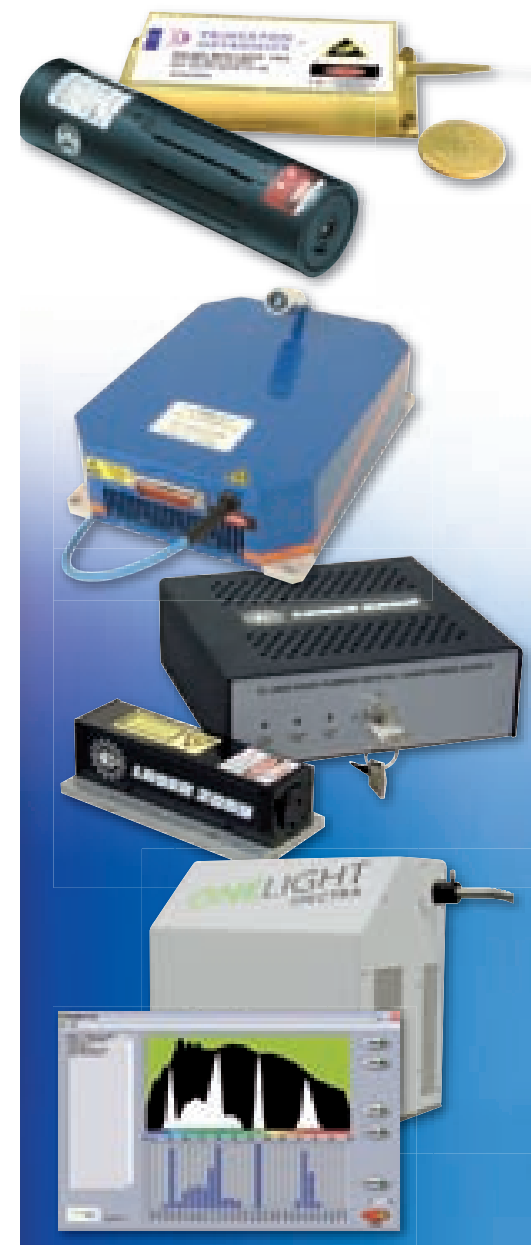

www.laser2000.fr 